\title{
Inflammatory Myofibroblastic Tumors in Childhood
}

\author{
A Report From the Italian Cooperative Group Studies
}

Rita Alaggio, MD'; Giovanni Cecchetto, MD²; Gianni Bisogno, MD³; Claudio Gambini, MD ${ }^{4}$; Maria Luisa Calabrò, $\mathrm{PhD}^{5}$; Alessandro Inserra, MD ; Renata Boldrini, MD7; Gian Luca De Salvo, MD*; Emanuele S. G. d'Amore, $M D^{9}$; and Patrizia Dall'Igna, $M D^{2}$

BACKGROUND: Inflammatory myofibroblastic tumors (IMTs) are myofibroblastic lesions with unpredictable biologic behavior that occur at a young age. For this report, the authors investigated clinicopathologic features in a series of pediatric IMTs. The objective of the study was to identify morphologic or immunohistochemical prognostic markers and the possible pathogenic role of human herpes virus 8 (HHV-8). METHODS: Twenty-six patients were observed over a period of 18 years. Clinical/histologic data were reviewed, and immunohistochemical/molecular studies were performed. RESULTS: Patients ages 8-216 months (median age, 60 months) presented with tumors of the lung-bronchus ( 8 patients), abdomen (17 patients), and thoracic wall (1 patient). Twenty-one patients underwent complete excision, and microscopic or macroscopic residual disease was present in 5 of those patients. Chemotherapy was received by 5 patients. After a median follow-up of 6.6 years, 24 patients were in complete remission, and 2 patients had died of disease. Local recurrences were observed in 6 patients (including 4 recurrences that occurred after a complete excision). Cytologic atypia, low inflammatory infiltrate, and a rich myxoid pattern were detected in patients who had recurrent disease or a poor prognosis. Anaplastic lymphoma kinase (ALK) was positive in 7 patients (including 2 patients with recurrent disease). No correlation between clusterin expression and prognosis was demonstrated. HHV-8 was identified in 1 pulmonary IMT. CONCLUSIONS: IMTs are locally aggressive lesions. In this series, the local recurrence rate was $23 \%$, and the 5-year and 10 -year event-free survival rates were $87.4 \%$ and $72.8 \%$, respectively. The results indicated that the treatment of choice is a complete, nonmutilating excision; chemotherapy may be given to patients who have microscopic or macroscopic residual disease, although the results are controversial; cytologic atypia and positive ALK status are more frequent in aggressive tumors, whereas metastatic tumors are negative for ALK; and HHV8 is not involved in the pathogenesis of IMT. Cancer 2010;116:216-26. (C) 2010 American Cancer Society.

KEYWORDS: inflammatory myofibroblastic tumors, anaplastic lymphoma kinase, clusterin, pseudotumor, children.

Inflammatory myofibroblastic tumors (IMTs) are a group of rare neoplastic lesions that occur in children and young adults. ${ }^{1}$ They may arise in any site, but the most frequent locations are the lungs, soft tissues, and the abdominal region. ${ }^{1,2}$ In $15 \%$ to $30 \%$ of patients, IMT can be associated with a clinical syndrome. ${ }^{3,4}$ The extrapulmonary forms have been reported in association with Wilms tumor, ${ }^{5}$ chronic sclerosing cholangitis, ${ }^{6}$ Hodgkin lymphoma, ${ }^{7}$ acute myelomonocytic leukemia, ${ }^{8}$ small cell carcinoma of the lung, ${ }^{7}$ and adrenocortical tumors. ${ }^{9}$ IMTs tend to recur locally and have a small risk of distant metastasis.

Corresponding author: Rita Alaggio, MD, Pathology Department, University Hospital of Padova, Via A. Gabelli, 61,35128 Padova, Italy; Fax: (011) 39 049 8272265; rita.alaggio@gmail.com

${ }^{1}$ Pathology Department, University Hospital of Padova, Padova, Italy; ${ }^{2}$ Pediatric Surgery Department, University Hospital of Padova, Padova, Italy; ${ }^{3}$ Pediatric Hematology-Oncology Department, University Hospital of Padova, Padova, Italy; ${ }^{4}$ Pathology Department, Giannina Gaslini Hospital of Genova, Genova, Italy; ${ }^{5}$ Immunology and Diagnostic Molecular Oncology, Veneto Oncology Institute, University of Padova, Padova, Italy; ${ }^{6}$ Pediatric Surgery Department, Bambino Gesù Hospital, Rome, Italy; ${ }^{7}$ Pediatric Pathology Department, Bambino Gesù Hospital, Rome, Italy; ${ }^{8}$ Clinical Trials and Biostatistics Unit, Veneto Oncology Institute, University of Padova, Padova, Italy; ${ }^{9}$ Pathology Department, San Bortolo Hospital of Vicenza, Vicenza, Italy

Presented at the 34th Meeting of the International Society of Pediatric Surgical Oncology-International Society of Pediatric Oncology, Porto, Portugal, September 18-21, 2002.

We thank the pediatric oncologists, pediatric surgeons, and pathologists who contributed to the article by referring patients from their institutions: Marco Forni, MD (Pathology Department, University-Hospital of Turin, Turin, Italy); Andrea Di Cataldo, MD (Pediatric Hematology-Oncology Department, University Hospital of Catania, Catania, Italy); and Giulio Murgia, MD (Pediatric Hematology Department, Regional Hospital for Microcytemia of Cagliari, Cagliari, Italy).

DOI: 10.1002/cncr.24684, Received: January 24, 2009; Revised: March 23, 2009; Accepted: March 31, 2009, Published online October 22, 2009 in Wiley InterScience (www.interscience.wiley.com) 
To prevent local recurrence, the treatment of choice for IMT is surgical excision. Chemotherapy also is used when a complete excision cannot be achieved. Moreover, cases of spontaneous remission after an incomplete excision, which was followed by steroids or nonsteroidal anti-inflammatory (NSAID) therapy, also have been reported. $^{10-12}$

The etiology of IMTs, their behavior, and their cell of origin remain matters of debate. ${ }^{13}$ Originally considered lesions with a benign clinical course, it is now clear that IMTs can have an aggressive behavior and, occasionally, an unfavorable prognosis. ${ }^{3}$ Both the "benign" and "malignant" forms share similar morphologic features, which are not predictive of prognosis or etiology, characterized by fascicles of bland myofibroblasts admixed with a prominent inflammatory component. ${ }^{1,2}$ Many viral agents, such as Epstein-Barr virus (EBV) and, more recently, human herpes virus 8 (HHV-8), have been implicated in the etiology of IMT. ${ }^{14,15}$

The chromosomal rearrangements that involve the anaplastic lymphoma kinase (ALK) gene locus in the chromosome $2 \mathrm{p} 23$ with other partner genes (tropomyosin 3 [TPM3] in chromosome 1 at $17 \mathrm{q} 23$ or TPM4 in chromosome 19 at $19 \mathrm{p} 13$ ) have been detected in $35 \%$ to $60 \%$ of IMTs, confirming the neoplastic nature of these lesions. ${ }^{16,17}$ The chimeric protein that results from the molecular rearrangement of the ALK gene can be detected by immunohistochemistry using the ALK-1 monoclonal antibody, which reveals specific staining patterns according to the different transcripts. The possible prognostic significance of ALK expression is controversial. Numerous studies investigating morphologic, immunohistochemical, and biomolecular prognostic markers to develop a more tailored treatment have been unsuccessful to date. ${ }^{1,2,16-18}$ In the current study, a series of pediatric IMTs were investigated to determine the relation between their clinicopathologic features and prognosis. The expression of ALK and the pathogenetic role of HHV-8 also were taken into account to determined whether they have a possible link with various clinical and histologic features.

\section{MATERIALS AND METHODS}

Patients' data were collected retrospectively through forms that were sent to the Italian Centers of Pediatric Surgery and Oncology. The forms required the permission of all participants in the study and the name of the physician responsible for each patient. The forms included information on clinical characteristics, treat- ment, outcome, and all data regarding tumor histology. Moreover, formalin-fixed, paraffin-embedded specimens were obtained from all patients.

\section{Histology}

All available hematoxylin and eosin-stained sections were reviewed by 2 pathologists, and representative blocks were selected for immunohistochemical and biomolecular studies. For each patient, the following histologic parameters were analyzed: 1) Cellularity and inflammatory infiltrate was classified as low, moderate, or high; 2) the presence of myxoid component and necrosis was classified as absent, focal (if they were identified in rare microscopic fields), or diffuse (when they involved most of the slides examined); 3) the number of mitoses per 10 high-power fields; and 4) the presence of mild, moderate, or severe nuclear atypia was classified as either focal (when isolated atypical cells were present) or diffuse (when atypical cells were numerous and appeared in different microscopic fields).

\section{Immunohistochemistry}

All available immunostainings were reviewed, and additional immunostainings were performed. The primary antibodies and antigen retrieval methods that were used for this study are indicated in Table 1 . The slides were incubated with the primary antibodies at room temperature for 1 hour. The immunoreaction detection was performed using either the LSAB+ kit (Dako, Glostrup, Denmark), which contains a secondary biotinylated antibody and streptavidin labeled with peroxidase, or the peroxidase-based EnVision kit (Dako), which is based on a unique enzyme-conjugated polymer backbone that carries secondary antibody molecules. 3,3'-Diaminobenzidine was used as the chromogen, and hematoxylin was used as a counterstain. The immunoreaction was considered positive when $\geq 5 \%$ of the neoplastic cells had unequivocal staining. Latency-associated nuclear antigen 1 (LANA-1) was positive if a strong, diffuse nuclear staining was evident in $>10 \%$ of cells.

\section{Polymerase Chain Reaction Analyses}

DNA was extracted from 10 specimens, and the quality of the DNA samples was tested by polymerase chain reaction (PCR) using primers specific for the $p 53$ gene. To detect the HHV-8 sequences, amplifications were performed by nested PCR using previously described HHV-3 (ORF25)-specific and HHV-8P (ORF26)-specific primers. ${ }^{19}$ Samples from the remaining children were not 
Table 1. Primary Antibodies/Antigen Retrieval Methods for Immunohistochemistry Studies

\begin{tabular}{lll} 
Antibody* & Dilution & Treatment \\
CD21 & $1: 50$ & - \\
CD23 & $1: 100$ & - \\
Clusterin & $1: 100$ & - \\
ALK-1 & $1: 200$ & Microwave \\
LANA-1 & $1: 500$ & Microwave \\
\hline
\end{tabular}

ALK-1 indicates a monoclonal antibody that recognizes the native anaplastic lymphoma kinase, LANA-1, latency-associated nuclear antigen 1.

*The source of all antibodies listed was Dako (Glostrup, Denmark).

analyzed by nested PCR, because the quantity was insufficient to allow for an accurate HHV-8 molecular analysis.

\section{Statistical Analysis}

Progression-free survival (PFS) was estimated using the Kaplan-Meier method and was calculated from the date of surgery to the date of disease recurrence, disease progression, death, or last follow-up if the patient remained alive in remission. The 5-year PFS rate was estimated along with the $95 \%$ confidence interval $(95 \% \mathrm{CI})$. Univariate survival analysis was performed using the log-rank test. All $P$ values were 2 -sided, and $P$ values $<.05$ were considered statistically significant. The SAS software package (version 9.1.3; SAS Institute, Inc., Cary, NC) was used for data analysis. The data were analyzed as of October 2008.

\section{RESULTS}

\section{Clinical Data}

Between January 1986 and December 2004, 26 patients (13 boys, 13 girls) with a diagnosis of IMT were treated in 7 Italian Pediatric Surgery and Oncology Centers. The patients' clinical characteristics are summarized in Table 2.

The patients ranged in age at presentation from 8 months to 216 months (median, 60 months). The lesions were located in the lung-bronchus in 8 patients, the thoracic wall in 1 patient, and the abdomen in 17 patients (including 11 patients with lesions in the mesenterybowel, 2 patients with lesions in the pelvis-bladder, 2 patients with lesions in the retroperitoneum, and 2 patients with lesions in the spleen and liver). One patient had been treated previously for Hodgkin lymphoma, and 3 patients had associated Fanconi disease, rheumatoid arthritis, and congenital immunodeficiency syndrome involving Fas-ligand deficit.

Symptoms at presentation varied according to the tumor site. Patients who had intrapulmonary lesions man- ifested mild respiratory symptoms or even distress; whereas patients who had abdominal tumors presented mostly with abdominal pain, urinary symptoms, and a palpable mass. Fever and inflammatory manifestations (increased C-reactive protein levels and white blood cells) were documented in 17 patients (6 patients with lesions in the lung-bronchus, 10 patients with lesions in the mesentery, and 1 patient with a lesion in the liver).

\section{Complete Excision}

Twenty-one patients underwent complete excision of the tumor: Twenty of those 21 patients did not receive any further treatment, and 1 patient who had tumor of the pelvis-bladder received chemotherapy (1 course of combined carboplatin, epirubicin, vincristine, actinomycin$\mathrm{D}$, ifosfamide, and etoposide [CEVAIE]). In 5 children, the mass was removed together with a bowel segment (the colon in 3 patients and the jejunum in 2 patients) to obtain a macroscopically radical excision. Seventeen patients were in first complete remission at a follow-up of 30 months to 160 months. Three patients were in second complete remission after a local recurrence; 1 of those patients underwent surgery alone, and the other 2 patients received chemotherapy and underwent second surgery. One of 21 patients who had tumors of the lung presented with a local recurrence and distant metastases in the brain 1 year after diagnosis and died of disease soon thereafter.

\section{Excision With Microscopic Residual Disease}

Two patients, a boy aged 3 years who had an IMT in the lung-bronchus and a boy aged 17 years who had a mesentery/jejunum lesion, underwent excision with microscopic residual tumor at diagnosis. The first patient, who had his tumor removed by bronchoscopy at diagnosis, underwent a right thoracotomy as a second surgical procedure to remove the macroscopic residual disease. The second patient, who had part of the jejunum excised, received vinblastine and methotrexate. Both patients remained alive with no evidence of disease at 144 months and 93 months, respectively, after their initial diagnosis.

\section{Initial Biopsy/Macroscopic Residual Disease}

One patient, a girl aged 12 years who had a lesion that involved the mesentery and pancreatic tail, underwent a second-look operation after chemotherapy (etoposide), but she manifested local recurrence and lung metastases and died of progressive disease 9 months after diagnosis. A girl aged 30 months who had a tumor of the pelvis-bladder received chemotherapy (CEVAIE) after undergoing 


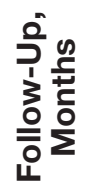

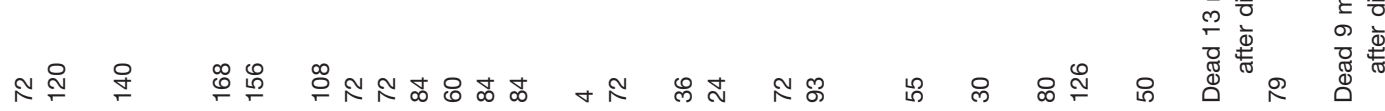

苞
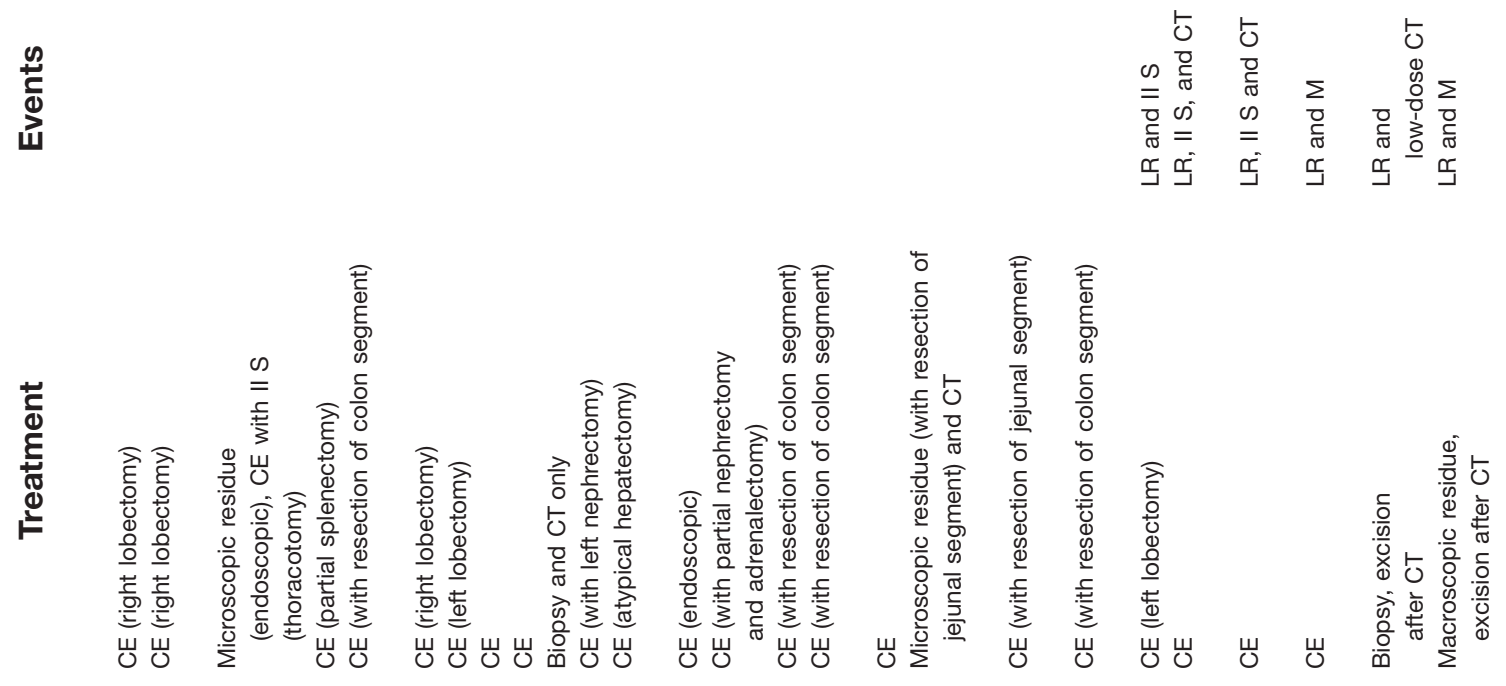

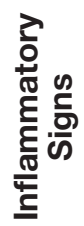

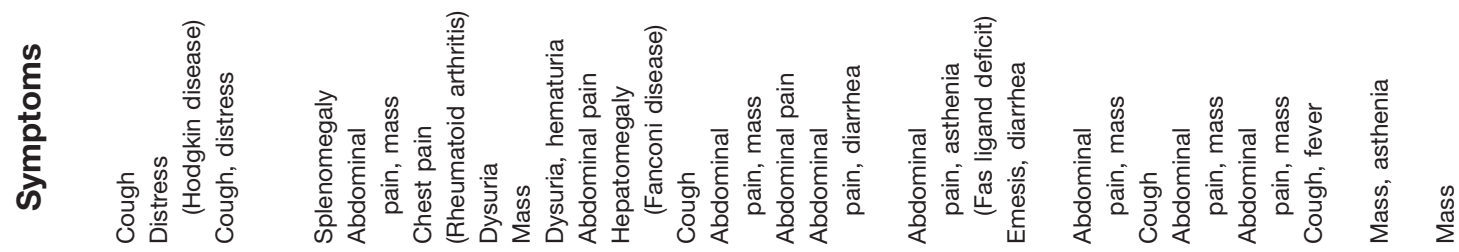
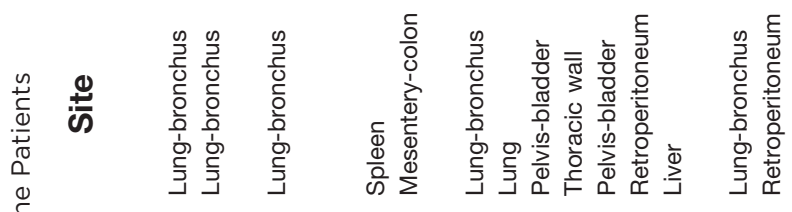

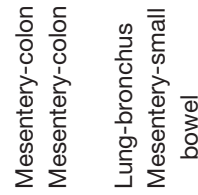
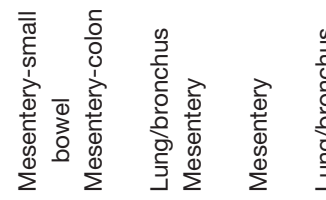

बำ

$\stackrel{+10}{a}$

$\infty$

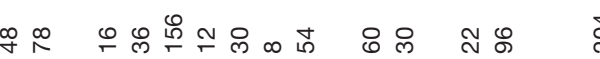

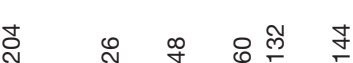

Z $\quad \stackrel{5}{A} \quad$

ふ 希雭

蒿 $-a$ $\checkmark$

수으도

$\stackrel{m}{\leftarrow} \stackrel{ }{\leftarrow} \stackrel{ }{\leftarrow}$

으 ำ

ก $\stackrel{\text { ก }}{\text { ก }}$ 


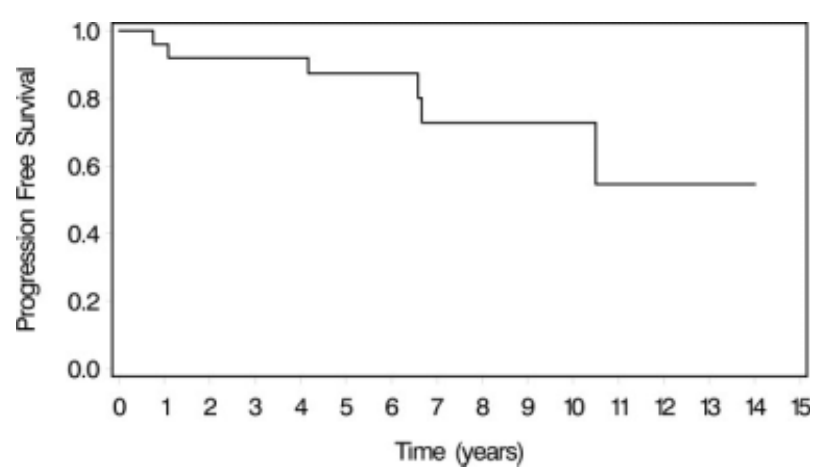

Figure 1. This chart illustrates 5-year and 10-year progressionfree survival for all patients.

biopsy and did not receive any other surgical treatment: She was alive and well 60 months after diagnosis. Another patient, a boy aged 12 years who had a huge tumor of the pelvis, received 1 cycle of chemotherapy (combined ifosfamide, vincristine, and actinomycin-D) after the initial biopsy and underwent a second surgical procedure with minimal residual disease. He manifested a local recurrence but, because of a previous strong toxicity, received low doses of methotrexate and NSAIDs. He was doing well 92 months after diagnosis.

After a median follow-up of 6.6 years, 20 patients remained alive in first complete remission, 4 patients remained alive in second complete remission, and 2 patients had died of disease. The estimated 5-year and 10-year PFS rates for all patients were $87.4 \%$ (95\%CI, 65.7-95.8\%) and $72.8 \%$ (95\%CI, 44.7-88.3\%), respectively (Fig. 1). On univariate analysis, the PFS rates were worse for patients who presented with cytologic atypia (log-rank test; $P=$ .007). Other histologic variables that were considered in the analysis did not have any statistically significant association with outcome, although patients who presented with tumors that had a myxoid component developed recurrent disease more frequently $(\log$-rank test; $P=.067)$.

\section{Histology}

In all patients, the tumors had a firm consistency, and their size ranged between $0.5 \mathrm{~cm}$ and $16 \mathrm{~cm}$ in greatest dimension (medium size, $6.5 \mathrm{~cm}$ ). Intra-abdominal IMTs in most patients were multinodular, noncapsulated, huge masses that adhered to and sometimes infiltrated the intestinal wall. On cross-section, the surface was whitish with firm, fleshy, or myxoid areas. In some patients, necrotic and hemorrhagic changes also were observed.

Pulmonary IMTs were characterized by various features. In 2 patients, the lesion was a single nodule that protruded into the lumen of the bronchus, infiltrating the wall and the surrounding pulmonary parenchyma. In the others, the lesion involved the lung parenchyma without involving the bronchus.

The majority of lesions were characterized by moderate (11 patients) to high (12 patients) cellularity and a prominent, inflammatory infiltrate that also was moderate to high. Fasciitis-like areas, which had elongated or plump myofibroblasts intermixed with numerous inflammatory cells, were combined in different proportions with myofibroma-like areas, which exhibited densely packed myofibroblasts in short fascicles with a minor inflammatory component. In 3 abdominal IMTs, a hypocellular pattern with fibrosis and poor inflammatory infiltrate was present (Fig. 2). Scattered or numerous cells in different microscopic fields with mild or moderate nuclear atypia and occasional ganglion-like features were detected in 3 thoracic tumors ( 1 in the thoracic wall) and in 5 abdominal tumors. A focal or diffuse myxoid background was typical in 7 abdominal and pelvic IMTs (Fig. 3). Necrosis was present in 2 abdominal tumors and in 2 thoracic tumors.

\section{Immunohistochemistry}

The typical myofibroblastic phenotype was confirmed in all patients by the immunostains. The tumors had diffuse, strong positivity for vimentin, and focal staining for smooth muscle actin was present in 24 tumors. The tumors were negative for desmin, S100, and the follicular dendritic cell markers CD21 and CD23. Clusterin exhibited a variable positivity, from weak to strong, in 14 of 26 tumors (53.8\%), including 6 tumors located in the lungs and 20 tumors in intra-abdominal locations (Fig. 4). The 2 intraparenchymal lesions (splenic and hepatic) were negative for clusterin.

\section{ALK Staining}

Positivity for ALK, which was characterized by a diffuse cytoplasmic immunostaining in myofibrolastic cells, was detected in 7 of the 26 tumors (27\%) and generally was more evident in the atypical and ganglion-like elements (Fig. 5). ALK positivity was demonstrated in 5 of 14 patients who had abdominal IMTs and in patients who had lesions of the liver and thoracic wall. LANA-1 immunostaining was performed in 20 patients to evaluate the status of HHV-8; the results were negative in 19 patients and positive in 1 patient (Patient 2).

\section{PCR Analyses}

Ten samples were analyzed for the presence of HHV-8 sequences by nested PCR (6 pulmonary tumors and 4 
abdominal tumors). All samples were negative for HHV8 except for 1 pulmonary IMT (Patient 2), which harbored sequences that were amplified from 2 nonoverlapping regions of the HHV-8 genome and expressed LANA-1 in myofibroblastic cells. The main histologic and immunohistochemical features are summarized in Table 3.

\section{DISCUSSION}

Originally described in the lung, ${ }^{2,20,21}$ IMTs have been reported in a variety of extrapulmonary sites and are most frequent in the mesentery, bowel, and genitourinary tract $^{1}$; the involvement of spleen and liver also has been described. ${ }^{22-24}$ Regardless of the site of origin, IMTs have been observed mainly in children and young adults. ${ }^{2,25-27}$

A palpable mass that mimics a malignancy may be the clinical presentation of these lesions; however, sometimes, they are discovered after the occurrence of an inflammatory syndrome associated with an inflammatory response at laboratory examination (microcytic hypochromic anemia, thrombocytosis, polyclonal hyperglobulinemia). ${ }^{1,28}$ In our group of patients, the lesions in the bronchus and lung were accompanied by nonspecific respiratory symptoms, whereas abdominal IMTs were associated with inflammatory signs and symptoms related to their local spread. It is noteworthy that 4 of our patients had an associated disease: Two girls were diagnosed with Fanconi anemia and rheumatoid arthritis, 1 boy was affected by a congenital immunodeficiency syndrome involving Fas-ligand deficit, and 1 girl had been treated for Hodgkin lymphoma before the occurrence of IMT. In the latter patient, in addition to chemotherapy, radiotherapy was delivered to the cervical, axillary, and mediastinal lymph nodes, but it is questionable whether the treatment may have been responsible for developing the IMT.

The clinical behavior of IMTs may be unpredictable. In most cases, they are benign lesions; however, occasionally, they are regarded as true neoplasms with a tendency for local recurrence and sometimes metastases. ${ }^{17}$ The treatment of choice for IMTs is surgical excision, because the response to chemotherapy and radiotherapy is variable. $^{3,29-31}$ In the current series, chemotherapy was successful in 3 of 5 patients at diagnosis, and 1 patient achieved a complete response. The remaining 2 patients did not respond to chemotherapy.

It has been well-documented that the malignant behavior of IMTs is observed mostly in patients who have abdominal tumors and undergo incomplete excision. ${ }^{32,33}$ The reported incidence of local recurrence in children
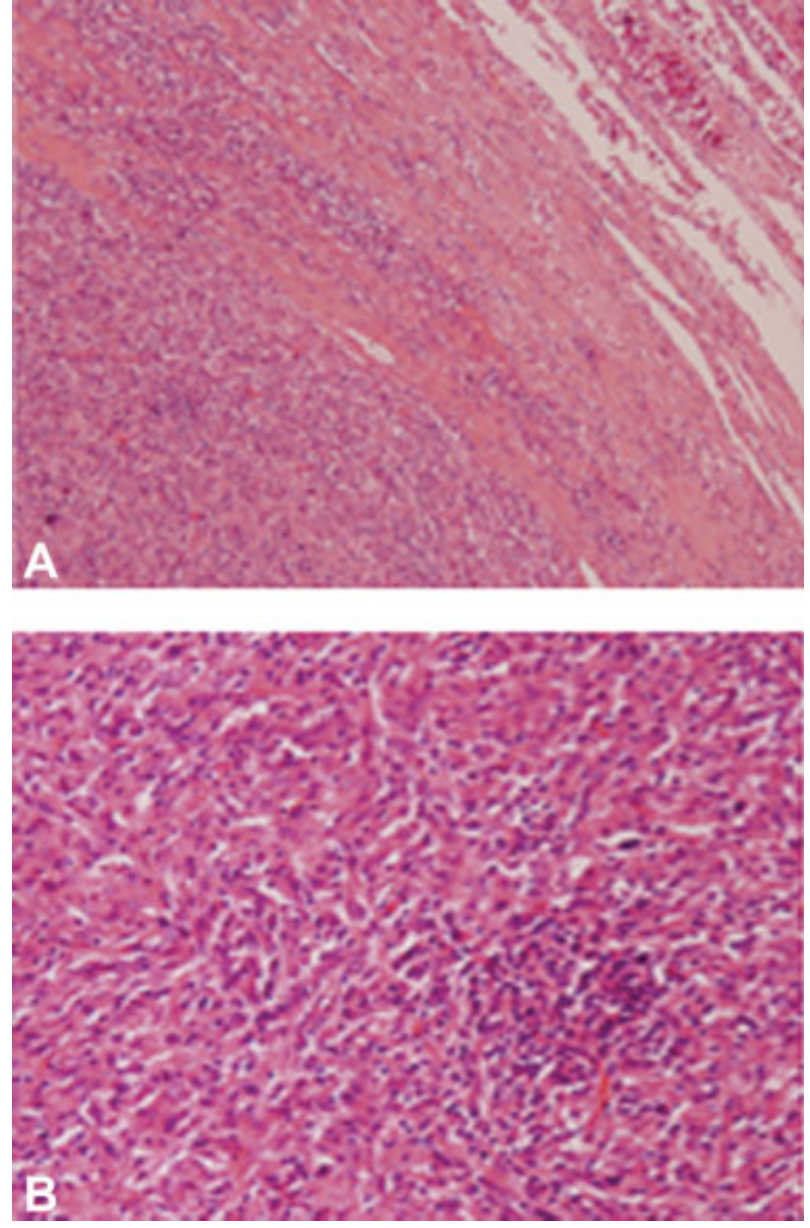

Figure 2. In Patient 24, who died of disease, photomicrographs of a pulmonary inflammatory myofibroblastic tumor revealed that the lesion (A) was well circumscribed and (B) demonstrated elongated cells intermixed with a low inflammatory infiltrate.

with mesentery and retroperitoneal tumors is between $15 \%$ and $37 \%,{ }^{3,29}$ and similar results were observed in our patients, who had an overall recurrence rate of $23 \%$. The recurrences developed in 4 of 6 patients who underwent radical surgery and in 2 patients who underwent biopsy as a primary procedure and had macroscopic residual disease. It may be hypothesized that, in the first 4 patients, the surgical excision had not been as complete as originally supposed and that chemotherapy had not been completely effective in the other patients. Conversely, 1 patient remained alive and disease free after chemotherapy and incomplete surgery. Moreover, chemotherapy was able to control the disease in 4 patients. It was associated with a second surgery and NSAID therapy in 3 patients and was the only treatment in 1 patient. It would be 

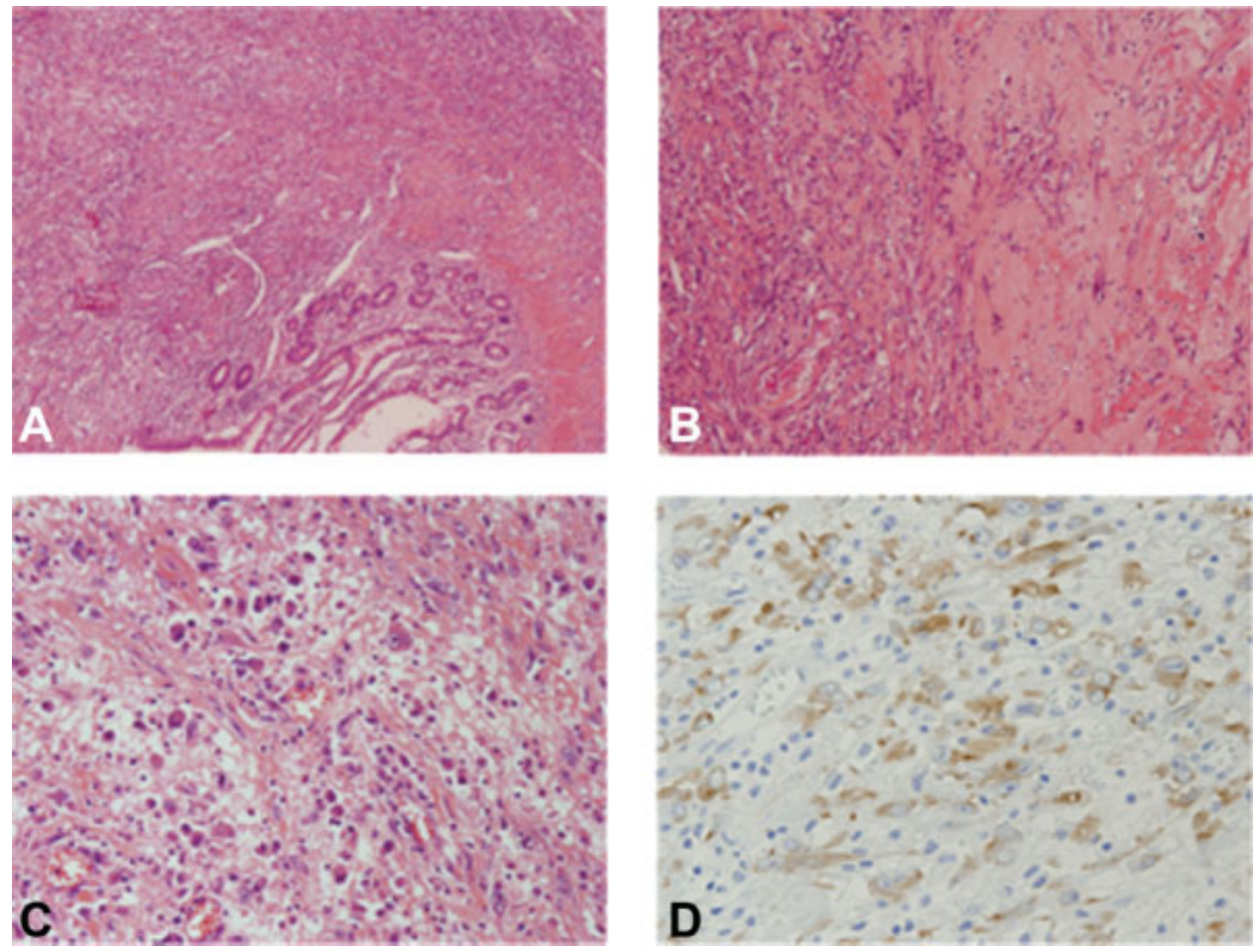

Figure 3. In Patient 25, photomicrographs of an abdominal inflammatory myofibroblastic tumor revealed that (A) the spindle cell proliferation involved the bowel wall, (B) myxoid areas were focally highly cellular with (C and D) typical ganglion-like cells, and (D) the cytoplasm was stained positive for anaplastic lymphoma kinase.

interesting to determine whether NSAIDs may have had some effect and whether the huge tumor that extended to the pelvis and bladder in the latter patient, who received chemotherapy only, underwent spontaneous regression. According to reports in the literature, a few patients have been treated successfully with chemotherapy using the regimens adopted for soft tissue sarcomas, but their role remains unproven. ${ }^{31}$ Regarding NSAIDs, many authors have suggested their effect, ${ }^{10-12}$ in particular because of their anti-inflammatory and antiangiogenetic properties. ${ }^{34}$ However, in the current series, NSAIDs were used only occasionally. Two children in our series died of progressive disease despite radical surgery in 1 patient and chemotherapy in both patients. Whether the aggressive clinical course and the recurrences were related to different biologic and morphologic tumor features remains a controversial issue. The histologic features of IMTs have been investigated thoroughly to identify the morphologic markers of a poor prognosis. In a recent study, cytologic atypias did not appear to be related to an unfavorable clinical course. ${ }^{17}$ In the current series, except in 1 patient, all recurrent tumors displayed focal or diffuse cytologic atypias and a myxoid background. These morphologic fea- tures were more frequent in abdominal IMTs, which are known as clinically more aggressive. The association of cytologic atypia and mesentery involvement appeared to be a strong predictor of aggressive behavior, as confirmed by evidence of local recurrence in 4 of 5 abdominal IMTs. Inflammatory infiltrate and necrosis did not reveal any relevant differences. Conversely, thoracic IMTs had greater inflammatory infiltrate and a complete absence of myxoid background, both of which were associated with a favorable clinical course in all but 1 patient.

The identification of a consistent aberration of chromosome 2p23 in a subgroup of IMTs, as demonstrated by the positive immunostaining for ALK, has revealed a new potential prognostic marker. ${ }^{16}$ ALK staining is positive in $34 \%$ to $56 \%$ of patients and is more frequent at an early age and at the abdominal site. To our knowledge to date, its prognostic role has not been completely clarified. ${ }^{35,36}$ ALK-positive IMTs are characterized by a higher recurrence rate; however, truly malignant lesions have been reported as ALK negative. ${ }^{17}$ The percentage of ALK-positive tumors in the current series was slightly lower $(28 \%)$, perhaps because of the high number of thoracic IMTs. In the current study, $35 \%$ of abdominal tumors, including 

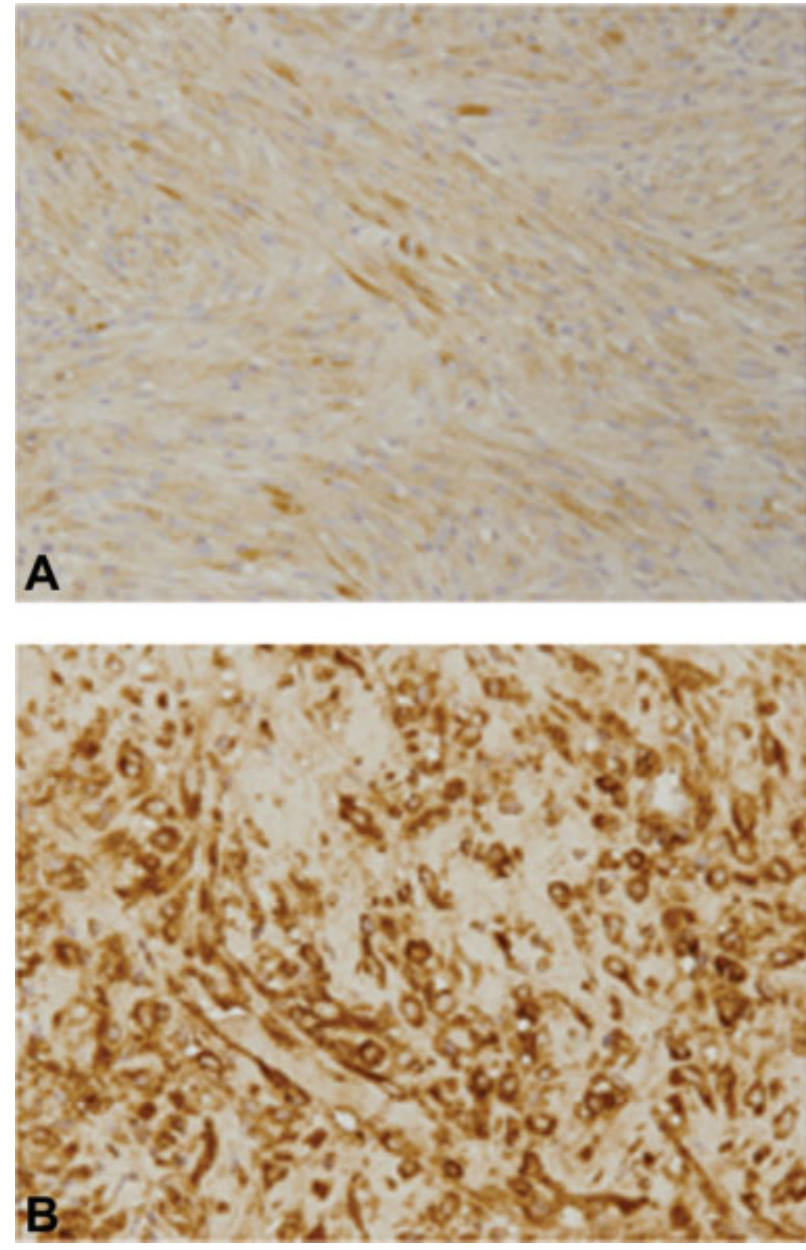

Figure 4. These photomicrographs revealed clusterin-positive immunostaining in 2 inflammatory myofibroblastic tumors of (A) the lung and (B) the urinary bladder.

the hepatic IMT and 1 IMT localized in the bladder, were positive for ALK, and 2 of them were associated with an immunologic or hematologic disorder. Only 1 extra-abdominal lesion was ALK positive. Regarding the recurrent tumors, they were more frequently ALK positive, but both tumors that had an unfavorable outcome were ALK negative. This apparent contradiction may be explained by the possible heterogeneity of the ALK-negative IMT, which may include different entities. Some may be true sarcomatous lesions, such as the aggressive lesions in our 2 patients who died. ${ }^{17}$ Other ALK-negative IMTs may represent inflammatory pseudotumors that most likely are related to viral infections. This fascinating hypothesis is supported by the association of the extra-abdominal IMT with some viral infections; in particular, both HHV-8 and EBV have been investigated as possible etiologic fac-
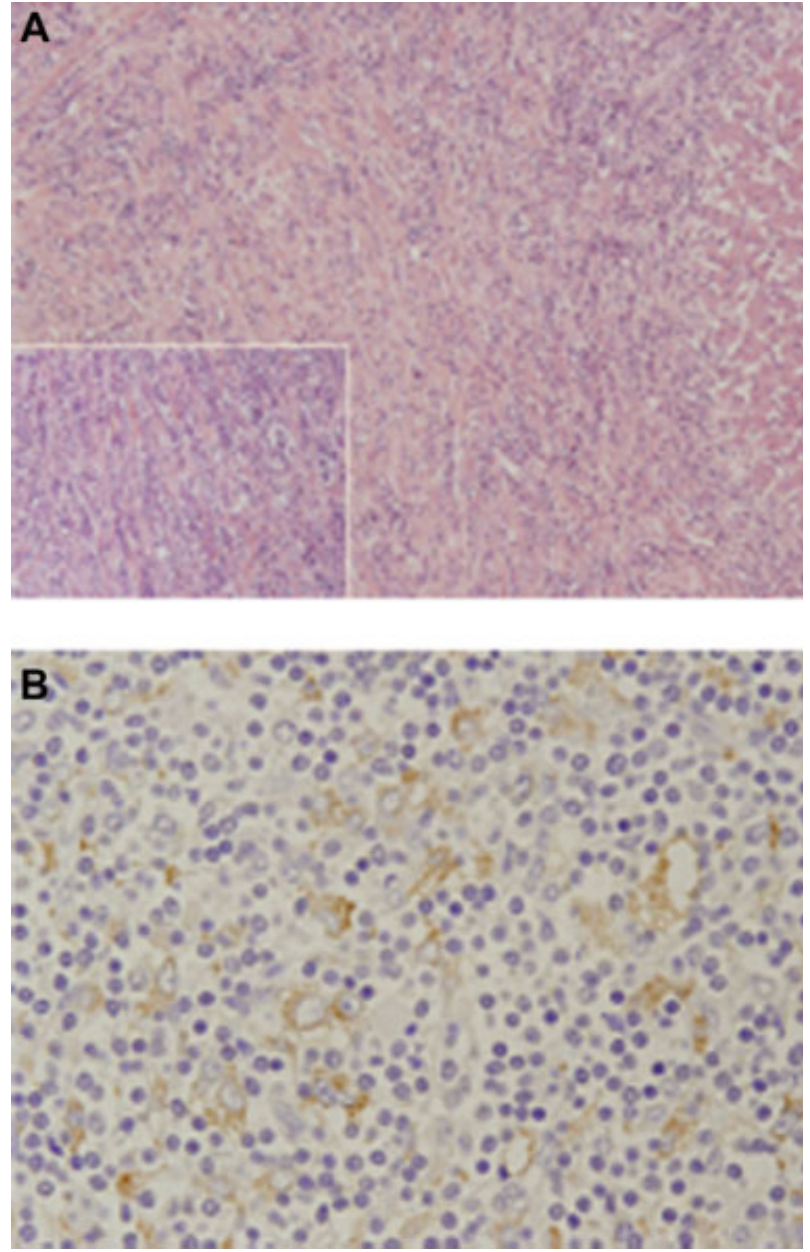

Figure 5. In Patient 12, photomicrographs of an inflammatory myofibroblastic tumor in the liver revealed (A) spindle cells with (inset) numerous inflammatory cells and (B) positive staining for anaplastic lymphoma kinase in scattered cells.

tors. ${ }^{9,10,27}$ The inflammatory signs and symptoms among the clinical manifestations seem to support an "infective" etiology. The possible pathogenetic role of HHV-8, which recently was postulated in pulmonary IMT in adults, ${ }^{18}$ is not confirmed in pediatric IMTs. In fact, similar to the series reported by Mergan et al, all but 1 of the IMTs in the current were negative for HHV-8. This detection rate ( 1 of 10 detected by nested-PCR) is in agreement with the reported seroprevalence of HHV-8 in Italian children, which ranges from $6 \%$ to $15 \%$, because Italy is a subendemic area for this virus. ${ }^{19,37,38}$ It is interesting to note that our HHV-8-positive tumor occurred in the patient who had a history of Hodgkin lymphoma, suggesting a possible pathogenetic role of immunodepression. Moreover, to the best of our knowledge, the role of 
$\underset{5}{\bar{j}}$

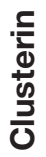

$\stackrel{\searrow}{\frac{1}{<}}$

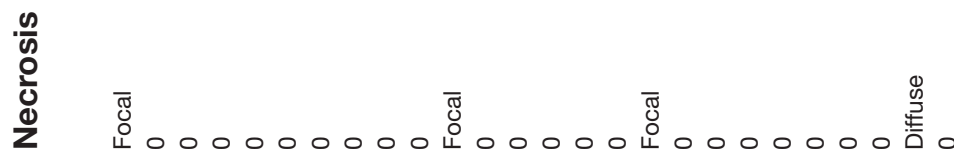

$\frac{\frac{1}{6}}{\overline{\frac{\pi}{3}}}$

$\frac{2}{\frac{8}{2}}$

券弯

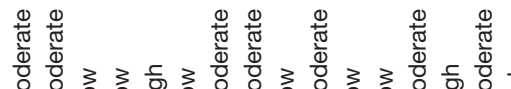

离

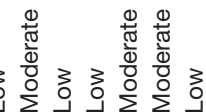

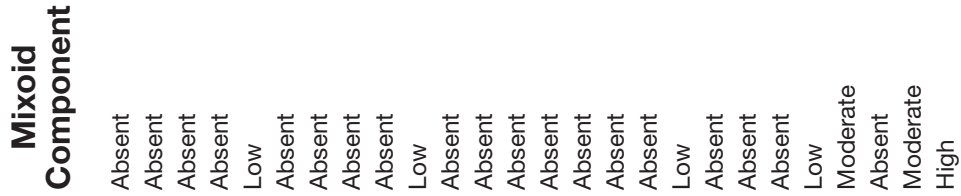

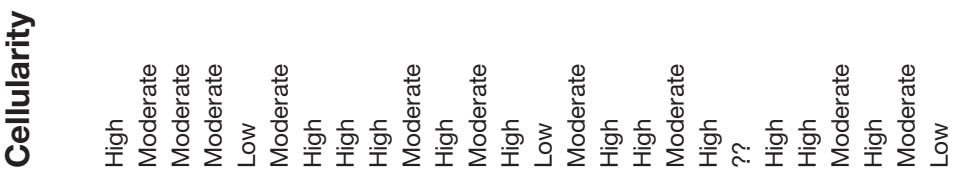

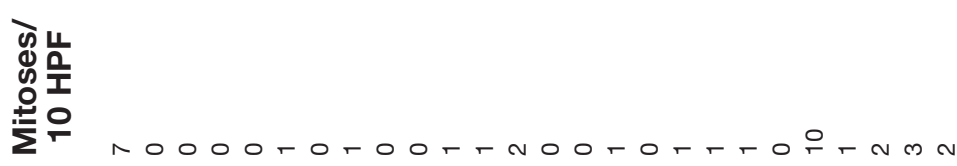

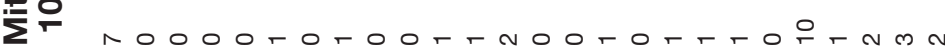

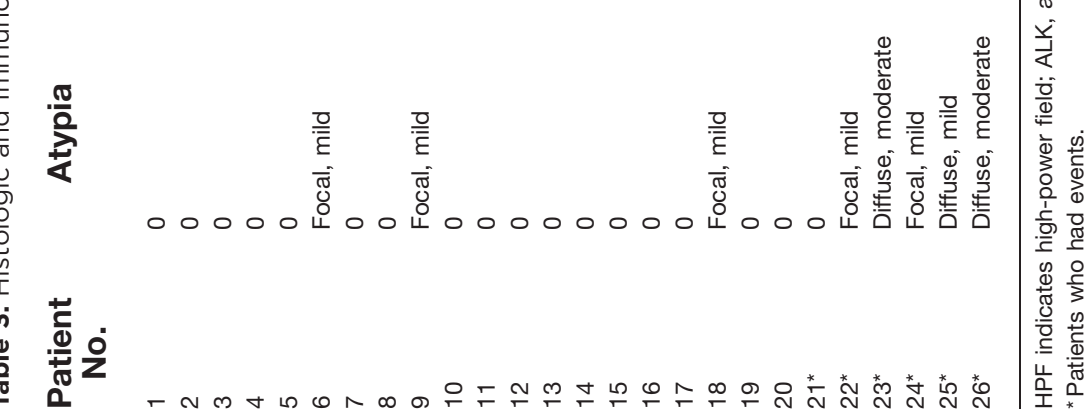


EBV in IMTs of the spleen, liver, and lymph nodes has not been established, ${ }^{39}$ and it has been suggested that this subgroup actually may represent follicular dendritic cell tumors (FDCTs). FDCTs typically are positive for CD21, CD23, and clusterin. In all of our patients, CD21 and CD23 invariably were negative, whereas positive cytoplasmic staining for clusterin was observed in 14 patients. Most of the lesions had only isolated cells, including 2 that were positive for ALK; however, in some lesions, the staining was strong and diffuse, mimicking the pattern observed in FDCTs. The positive staining for clusterin may represent a potential pitfall in the histologic differential diagnosis and emphasizes the importance of using a wide immunohistochemical panel, especially in small biopsies. Its significance remains unclear. Clusterin is an antiapoptotic protein that is released by fibroblasts in response to mechanisms that induce cellular damage. It is possible that fibroblasts and myofibroblasts express this protein in response to unknown pathogenetic mechanisms, such as viruses or other immunomediated reactions, as suggested by the more frequent positive staining for clusterin in lesions that have a rich inflammatory component or in lesions that occur in patients who have a previous history of lymphoma or immunitary disorders (3 of 4 lesions were clusterin positive).

In conclusion, in the current study, we reported a series of children with IMT who had a recurrence rate of $23 \%$, including 2 children who had an unfavorable prognosis despite a complete surgical excision being performed in 1 child. These data are far from conclusive but suggest that a cautious therapeutic approach should be taken to these lesions, and the main objective must be a complete excision that attempts to avoid mutilating surgery. In patients who have microscopic or macroscopic residual disease, a chemotherapy regimen similar to that used for soft tissue sarcomas may be used, even if the results are controversial. ${ }^{3,30}$ The histology of IMT cannot predict the clinical course; nevertheless, the abdominal site is associated with cytologic atypia, frequent ALK positivity, and a higher recurrence rate, confirming the neoplastic nature of this group of IMTs. The association of ALK-positive IMT with immunologic or hematologic disorders deserves further investigation. ALK-negative IMTs remain a conundrum. They include aggressive, sarcomatous lesions that respond poorly to surgery and chemotherapy and inflammatory pseudotumors with an infective etiology that are undistinguishable morphologically from true ALK-negative IMTs. The real incidence of true ALK-negative IMTs still is debated, and future stud- ies will contribute toward clarifying their nature. Nonetheless, the current results indicate that HHV-8 infection is not involved in pediatric IMT.

\section{CONFLICT OF INTEREST DISCLOSURES}

Supported in part by a grant from Fondazione "Città della Speranza," Fondazione Berlucchi and "Fondazione Cariparo" for the TREP Study (Tumori Rari in Età Pediatrica-Rare Tumors in Childhood).

\section{REFERENCES}

1. Coffin CM, Watterson J, Pries JR, et al. Extrapulmonary inflammatory myofibroblastic tumor (inflammatory pseudotumor). A clinicopathologic and immunohistochemical study of 84 cases. Am J Surg Pathol. 1995;19:859-872.

2. Coffin CM, Humphrey PA, Dehner LP. Extrapulmonary inflammatory myofibroblastic tumor: a clinical and pathologic survey. Semin Diagn Pathol. 1998;15:85-101.

3. Meis JM, Enzinger FM. Inflammatory fibrosarcoma of the mesentery and retroperitoneum. A tumor closely simulating inflammatory pseudotumor. Am J Surg Pathol. 1991;15: 1146-1156.

4. Gleason B, Hornick J. Inflammatory myofibroblastic tumor: were are we now? J Clin Pathol. 2008;61:428-437.

5. Newbould MJ, Kelsey A, Lendon M, et al. Inflammatory pseudotumor of the liver masquerading as a metastasis in a child treated for nephroblastoma. Med Pediatr Oncol. 1992; 20:172-175.

6. Gough J, Chakrabarti S. Inflammatory pseudotumor of the liver in a patient with chronic sclerosing cholangitis. Am J Gastroenterol. 1993;88:1452-1453.

7. Thomas RM, Jaffe ES, Zarate-Osorno A, et al. Inflammatory pseudotumor of the spleen. Arch Pathol Lab Med. 1993;117:921-926.

8. Isobe H, Nishi Y, Fukutomi T, et al. Inflammatory pseudotumor of the liver associated with acute myelomonocytic leukemia. Am J Gastroenterol. 1991;86:238-240.

9. $\mathrm{Fu} \mathrm{KH}$, Liu HW, Leung CY. Inflammatory pseudotumor of the spleen. Histopathology. 1990;12:302-304.

10. Doski JJ, Driessnack M, et al. Corticosteroids in the management of unresected plasma cell granuloma (inflammatory pseudotumor) of the lung. J Pediatr Surg. 1991; 26:10641066.

11. Su W, Ko A, O'Connel TX, et al. Treatment of pseudotumors with nonsteroids anti-inflammatory drugs. J Pediatr Surg. 2000;35:1635-1637.

12. Fletcher SG, Galgano MT, Michalski MP, et al. Regression of inflammatory pseudotumor of the bladder in a child with medical management [serial online]. Urology. 2007; 69:982.e11-982.e12.

13. Nonaka D, Birbe R, Rosai J. So-called inflammatory myofibroblastic tumor: a proliferative lesion of fibroblastic reticulum cells? Histopathology. 2005;46:604-613.

14. Gomez-Roman JJ, Ocejo-Vinyals G, Sanchez-Velasco P, Nieto EH, Leyva-Cobian F, Val-Bernal JF. Presence of human herpesvirus-8 DNA sequences and overexpression of human IL-6 and cyclin D1 in inflammatory myofibroblastic tumor (inflammatory pseudotumor). Lab Invest. 2000; 80:1121-1126. 
15. Ding Y, Saylors RL, Brown H, et al. Pulmonary inflammatory pseudotumor with HHV-8. Am J Surg Pathol. 2002;26:1089-1091; author reply 1091-1092.

16. Coffin CM, Patel A, Perkins S, et al. ALK1 and p80 expression and chromosomal rearrangements involving $2 \mathrm{p} 23$ in inflammatory myofibroblastic tumor. Mod Pathol. 2001; 14:569-576.

17. Coffin CM, Hornick JL, Fletcher CDM. Inflammatory myofibroblastic tumor. Comparison of clinicopathologic, histologic, and immunohistochemical features including ALK expression in atypical and aggressive cases. Am J Surg Pathol. 2007;31:509-520.

18. Mergan F, Jaubert F, Sauvat F, et al. Inflammatory myofibroblastic tumor in children: clinical review with anaplastic lymphoma kinase, Epstein-Barr virus, and human herpesvirus 8 detection analysis. J Pediatr Surg. 2005; 40:15811586.

19. Calabró ML, Gasperini P, Barbierato $M$, et al. A search for human herpesvirus 8 (HHV-8) in HIV-1 infected mothers and their infants does not suggest vertical transmission of HHV-8. Int J Cancer. 2000;85:296-297.

20. Brunn $H$. Two interesting benign tumors of contradictory histopathology. J Thorac Surg. 1939;9:119-131.

21. Umiker WO, Iverson L. Post-inflammatory tumors of the lung. J Thorac Surg. 1954;28:55-63.

22. Dall'Igna P, Cecchetto G, Guglielmi M, Alaggio R. Clinical and pathologic considerations in a case of inflammatory myofibroblastic tumor of the spleen. Pediatr Surg Int. 2004;20:875-877.

23. Aru GM, Abramowski CR, Ricketts RR. Inflammatory pseudotumor of the spleen in a young child. Pediatr Surg Int. 1997;12:299-301.

24. Dasgupta D, Guthrie A, McClean P, et al. Liver transplantation for a hilar inflammatory tumor. Pediatr Transplant. 2004;8:517-521.

25. Dewar AL, Connett GJ. Inflammatory pseudotumor of the trachea in a 10 month old infant. Pediatr Pulmonol. 1997;23:307-309.

26. Mynt MA, Medeiros LJ, Sulaiman RA, et al. Inflammatory pseudotumor of the ileum. Arch Pathol Lab Med. 1994;118:1138-1142.

27. Riedel BD, Wong RC, Ey EH, et al. Gastric inflammatory myofibroblastic tumor (inflammatory pseudotumor) in infancy: case report and review of the literature. J Pediatr Gastroenterol Nutr. 1994;19:437-443.
28. Karnak I, Senocak ME, Ciftci AO, et al. Inflammatory myofibroblastic tumor in children: diagnosis and treatment. J Pediatr Surg. 2001;36:908-912.

29. Souid AK, Ziemba MC, Dubanski AS, et al. Inflammatory myofibroblastic tumor in children. Cancer. 1993;72: 2042-2048.

30. Chun YS, Wang L, Nascimento AG, et al. Inflammatory myofibroblastic tumor: anaplastic lymphoma kinase (ALK) expression and prognosis. Pediatr Blood Cancer. 2005;45: 796-801.

31. Dishop MK, Warner BW, Dehner LP, et al. Successful treatment of inflammatory myofibroblastic tumor with malignant transformation by surgical resection and chemotherapy. J Pediatr Surg. 2003;25:153-158.

32. Morotti RA, Legman MD, Kerkar N, et al. Pediatric inflammatory myofibroblastic tumor with late metastasis to the lung: case report and review of the literature. Pediatr Dev Pathol. 2005;8:224-229.

33. Donner LR, Trompler RA, White RR, et al. Progression of inflammatory myofibroblastic tumor (inflammatory pseudotumor) of soft tissue into sarcoma after several recurrences. Hum Pathol. 1996;27:1095-1098.

34. Applebaum H, Kiera MK, Cripe TP, et al. The rationale for nonsteroidal anti-inflammatory drug therapy for inflammatory myofibroblastic tumor: a Children's Oncology Group Study. J Pediatr Surg. 2005;40:999-1003.

35. Cessna MH, Zhou H, Sanger WG, et al. Expression of ALK1 and p80 in inflammatory myofibroblastic tumor and its mesenchymal mimics: a study of 135 cases. Mod Pathol. 2002;15:931-938.

36. Cook JR, Dehner LP, Collins MH, et al. Anaplastic lymphoma kinase (ALK) expression in the inflammatory myofibroblastic tumor: a comparative immunohistochemical study. Am J Surg Pathol. 2001;25:1364-1371.

37. Calabró ML, Sheldon J, Bavero A, et al. Seroprevalence of Kaposi's sarcoma-associated herpesvirus/human herpesvirus 8 in several regions of Italy. J Hum Virol. 1998;1:207-213.

38. Cattani P, Cerimele F, Porta D, et al. Age-specific seroprevalence of human herpesvirus 8 in Mediterranean regions. Clin Microbiol Infect. 2003;9:274-279.

39. Kutok JL, Pinkus GS, Dorfman DL, et al. Inflammatory pseudotumor of lymph node and spleen: an entity biologically distinct from inflammatory myofibroblastic tumor. Hum Pathol. 2001;32:1382-1387. 